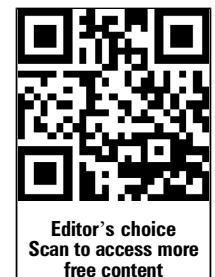

Memorial Hospital of Rhode Island and Brown University, Pawtucket, Rhode Island, USA

Correspondence to Dr F Dennis McCool, Pulmonary, Critical Care and Sleep Medicine, Memorial Hospital of Rhode Island and Brown University, Pawtucket, RI 02860,

USAF_McCool@Brown.edu

Received 2 July 2013

Revised 20 November 2013

Accepted 27 November 2013

Published Online First

23 December 2013

\section{SLinked}

http://dx.doi.org/10.1136/ thoraxjnl-2013-204920

\section{CrossMark}

To cite: DiNino $\mathrm{E}$ Gartman EJ, Sethi JM, et al. Thorax 2014;69:423-427.

\title{
Diaphragm ultrasound as a predictor of successful extubation from mechanical ventilation
}

\author{
Ernest DiNino, Eric J Gartman, Jigme M Sethi, F Dennis McCool
}

\begin{abstract}
Introduction The purpose of this study was to evaluate if ultrasound derived measures of diaphragm thickening, rather than diaphragm motion, can be used to predict extubation success or failure.

Methods Sixty-three mechanically ventilated patients were prospectively recruited. Diaphragm thickness (tdi) was measured in the zone of apposition of the diaphragm to the rib cage using a 7-10 MHz ultrasound transducer. The percent change in tdi between end-expiration and end-inspiration ( $\Delta$ tdi $\%)$ was calculated during either spontaneous breathing (SB) or pressure support (PS) weaning trials. A successful extubation was defined as SB for $>48 \mathrm{~h}$ following endotracheal tube removal.

Results Of the 63 subjects studied, 27 patients were weaned with SB and 36 were weaned with PS. The combined sensitivity and specificity of $\Delta$ tdi $\% \geq 30 \%$ for extubation success was $88 \%$ and $71 \%$, respectively. The positive predictive value and negative predictive value were $91 \%$ and $63 \%$, respectively. The area under the receiver operating characteristic curve was 0.79 for $\Delta$ tdi $\%$. Conclusions Ultrasound measures of diaphragm thickening in the zone of apposition may be useful to predict extubation success or failure during SB or PS trials.
\end{abstract}

\section{INTRODUCTION}

Timing is critical when determining if a patient can be successfully extubated. Premature discontinuation of mechanical ventilation may lead to increased cardiovascular and respiratory stress, $\mathrm{CO}_{2}$ retention and hypoxaemia with up to $25 \%$ of patients requiring reinstitution of ventilator support. ${ }^{1}{ }^{2}$ Unnecessary delays in weaning from mechanical ventilation also can be deleterious. Complications such as ventilator associated pneumonia and ventilator induced diaphragm atrophy can be seen with short periods of mechanical ventilation thereby prolonging mechanical ventilation. ${ }^{3-6}$

Tools available for determining the optimal timing of extubation are limited. Subjective decisions are often wrong. ${ }^{7}$ Stroetz and Hubmayr ${ }^{8}$ found that clinical prediction of extubation success or failure was often incorrect with the decision to extubate biased toward ventilator dependency. Measures such as breathing frequency (f), minute ventilation, and negative inspiratory force, have done little to improve the timing of successful extubation. ${ }^{9-11} \mathrm{~A}$ more recent parameter, the rapid shallow breathing index (RSBI) provides a guide for timing extubation with spontaneous breathing (SB) trials but its value is limited when used to predict successful extubation during pressure support (PS) trials. $^{12} 13$

\section{Key messages}

What is the key question?

- Can ultrasound measurements of diaphragm muscle thickening during inspiration provide a measure of extubation success or failure?

What is the bottom line?

- We found that measurements of diaphragm thickening in the zone of apposition outperform standard measures of extubation outcome.

\section{Why read on?}

- Learn the rationale for using ultrasound measures of diaphragm muscle thickening to predict extubation outcomes, how these measures differ from ultrasound measures of diaphragm dome motion, and how this measure compares with other measures used to predict extubation success or failure.

Direct measures of diaphragm function as predictors of extubation success or failure have not been extensively evaluated. Motion of the diaphragm dome has been assessed using $\mathrm{M}$ mode ultrasound ${ }^{14} 15$ and found to be useful in predicting extubation outcomes ${ }^{16}{ }^{17}$; however, imaging the dome does not directly visualise the diaphragm muscle itself and factors such as breath size, impedance of neighbouring structures, abdominal compliance, rib cage or abdominal muscle activity, and ascites will affect regional and global diaphragm motion of the tendonous dome of the diaphragm. ${ }^{18}$ This drawback can be circumvented by ultrasonography of the diaphragm in the zone of apposition (ZAP) as this approach allows for direct visualisation of the diaphragm muscle and assessment of its activity. ${ }^{19-25}$ Diaphragm thickening during inspiration reflects diaphragm shortening and is analogous to an 'ejection fraction' of the heart. The purpose of this study was to assess whether the degree of diaphragm thickening as measured by $\mathrm{B}$ mode ultrasound during a weaning trial can be used to predict extubation outcomes.

\section{METHODS}

\section{Subjects}

Sixty-three patients were prospectively recruited from medical intensive care units at Memorial Hospital of Rhode Island and Rhode Island Hospital. The study was approved by the 
institutional review boards at both the institutions (IRB \#09-45 for Memorial Hospital of Rhode Island and CMTT \#0095-10 for Rhode Island Hospital) and informed consent was obtained for all study participants. All patients were clinically stable and identified by the intensivist as ready to undergo a low-level PS weaning trial or a SB trial. Subjects were recruited 6-24 h prior to the first weaning trial. The ultrasonographer was notified of the intensivist's decision to start weaning and obtained informed consent. No specific disease process was required for entry into the study other than the presence of respiratory failure. All subjects had a fraction of inspired oxygen (FIO2) of $<50 \%$, were haemodynamically stable in the absence of vasopressors, and alert. Exclusion criteria included pregnant women, age $<18$ years and surgical dressings over the right lower rib cage which would preclude ultrasound exam. Subject characteristics are given in table 1 and the causes of respiratory failure are summarised in figure 1 .

\section{Measurements}

Diaphragm thickness (tdi) was measured using a $7-10 \mathrm{MHz}$ linear ultrasound probe set to B mode (LOGIQ Book, GE Healthcare, Waukesha, Wisconsin, USA). The right hemidiaphragm was imaged at the zone of apposition of the diaphragm and rib cage in the midaxillary line between the 8 th and 10th intercostal spaces as previously described. ${ }^{19}$ All patients were studied with the head of bed elevated between $20^{\circ}$ and $40^{\circ}$. The tdi was measured at end-expiration and end-inspiration. A flowmeter (Hamilton FlowSensor $\mathrm{H}$ ) was placed in the ventilator circuit and flow recorded simultaneously with ultrasound images to confirm end-expiration and end-inspiration on the ultrasound images. The per cent change in tdi between end-expiration and end-inspiration $(\Delta$ tdi $\%)$ was calculated as (tdi end-inspiration-tdi end-expiration/tdi end-expiration) $\times 100$. The $\Delta \mathrm{tdi} \%$ for each patient represented the mean of three to five breaths. Images were obtained within the first $5 \mathrm{~min}$ of the SB or the PS trial. Training the ultrasound operator to identify the diaphragm and measure its thickness took three to five sessions lasting 10-15 min each. Intraobserver variability in measuring $\Delta$ tdi $\%$ was $<10 \%$.

\section{Protocol}

Weaning trials consisted of SB trials (t-piece with zero PS) or PS trials (reducing PS by $5 \mathrm{~cm} \mathrm{H}_{2} \mathrm{O}$ until a PS level of $\Delta 5 / 5$ was obtained). The different types of weaning trials (SB vs PS) reflected different practice patterns among the intensivists caring

\begin{tabular}{|c|c|c|c|c|c|c|}
\hline $\begin{array}{l}\text { Weaning } \\
\text { index }\end{array}$ & Number & $\begin{array}{l}\text { Sensitivity } \\
(\%)\end{array}$ & $\begin{array}{l}\text { Specificity } \\
(\%)\end{array}$ & $\begin{array}{l}\text { PPV } \\
(\%)\end{array}$ & $\begin{array}{l}\text { NPV } \\
(\%)\end{array}$ & ROC \\
\hline \multicolumn{7}{|l|}{$\Delta \mathrm{tdi} \%$} \\
\hline$\geq 30 \%$ & 63 & 88 & 71 & 91 & 63 & 0.79 \\
\hline \multicolumn{7}{|l|}{$\Delta$ tdi $^{2} \times \mathrm{V}_{\mathrm{T}}$} \\
\hline$\geq 80$ & 62 & 88 & 71 & 91 & 63 & 0.76 \\
\hline \multicolumn{7}{|c|}{ tdi at end-expiration } \\
\hline$\geq 0.17 \mathrm{~cm}$ & 63 & 90 & 21 & 80 & 38 & 0.61 \\
\hline \multicolumn{7}{|l|}{$\Delta$ tdi/f } \\
\hline$\geq 0.008$ & 62 & 92 & 67 & 90 & 71 & 0.75 \\
\hline
\end{tabular}

Threshold for $\Delta$ tdi $\%=30 \%$; Threshold for $\Delta t d i \% \times V_{T}=80(\mathrm{ml} \%)$; Threshold for tdi at end-expiration $=0.17 \mathrm{~cm}$; Threshold for $\Delta \mathrm{tdi} \% / \mathrm{f}=0.008$ (\%/breaths $/ \mathrm{min})$.

NPV, negative predictive value; PPV, positive predictive value; ROC, receiver operating

characteristic; tdi, diaphragm thickness.

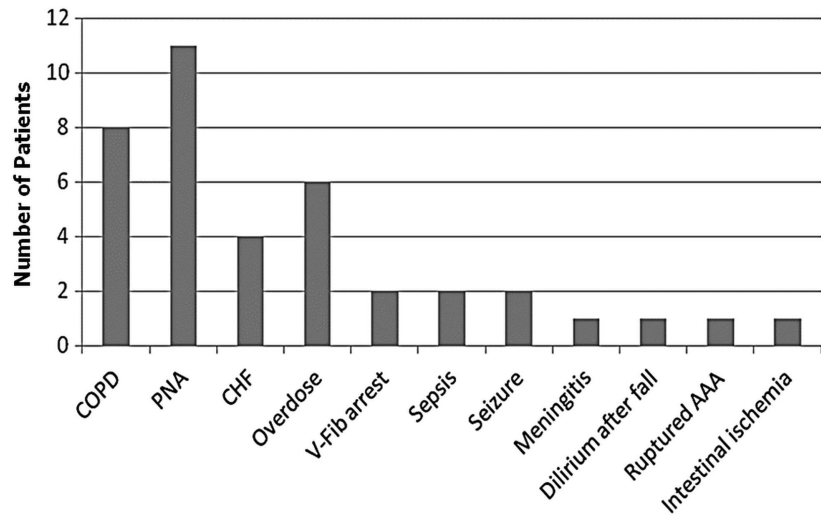

Figure 1 Aetiology of respiratory failure in the subject population.

for the patients. The treating team was blinded to the ultrasound results, and the research team did not have a role in deciding whether or not a patient was extubated. A successful extubation was defined as SB for $>48 \mathrm{~h}$ following extubation. A failed extubation was defined as someone who was reintubated within $48 \mathrm{~h}$, terminally extubated, or requiring tracheostomy. Most patients were extubated within 6-12 h of measuring tdi (48/63) and nine had measurements within $12-36 \mathrm{~h}$ of extubation. Of the remaining six, three were terminally extubated and three required tracheostomy. The RSBI ( $f$ in breaths/minute (f)/ tidal volume in litres $\left(\mathrm{V}_{\mathrm{T}}\right)$ ) was calculated simultaneously with ultrasound measurements of tdi only during SBT.

Measures of tdi at end-expiration have been shown to reflect diaphragm strength in healthy individuals. ${ }^{23}$ Since a sufficiently strong diaphragm would promote successful extubation, we evaluated whether tdi end-expiration would be useful for determining extubation success or failure. Since the diaphragm shortening contributes to tidal volume, we combined $\Delta$ tdi $\%$ with measures of breathing pattern $\left(\mathrm{V}_{\mathrm{T}}\right.$ and $\left.\mathrm{f}\right)$ and evaluated whether these parameters could predict extubation success or failure.

\section{Analysis}

Descriptive statistics are presented as the mean \pm SD for continuous variables. $\chi^{2}$ tests were used to assess differences in groups for the categorical variables. Sensitivity, specificity, positive predictive value (PPV) and negative predictive value (NPV) were calculated for ultrasound derived measurements of tdi in the PS and SB trial groups and for RSBI in the SB trial group in predicting extubation success. The cut-off points for each index are given in table 2. Receiver operating characteristic (ROC) curves were used to evaluate the efficacy of ultrasound derived measurements of $\Delta$ tdi $\%$, tdi end-expiration, $\Delta$ tdi $\% \times \mathrm{V}_{\mathrm{T}}, \Delta \mathrm{tdi} \% / \mathrm{f}$ and RSBI in predicting extubation success or failure. Median and IQR were calculated for ventilator days for the PS and SB trial groups. A Wilcoxon test was used to compare ventilator days between the PS and the SB trial groups. A p value $<0.05$ was considered statistically significant. Data analyses were performed using SAS V.9.2 (SAS Institute, Cary, North Carolina, USA).

\section{RESULTS}

Sixty-three patients were studied. Thirty-six patients $(17 \mathrm{men}$, 19 women; average age $66.0 \pm 19$ (SD) years had measurement of $\Delta$ tdi $\%$ at a PS of $\Delta 5 / 5$ and 27 patients ( 14 men, 13 women; average age $69.6 \pm 17$ years) had measurements of $\Delta$ tdi $\%$ during a SB trial. There was no difference in ventilator days between the PS and SB trial groups. $(p=0.97)$. The 36 patients weaned 
Table 2 Weaning parameters

\begin{tabular}{|c|c|c|c|c|c|}
\hline Weaning index & Number & $\begin{array}{l}\text { Sensitivity } \\
(\%)\end{array}$ & $\begin{array}{l}\text { Specificity } \\
(\%)\end{array}$ & $\begin{array}{l}\text { PPV } \\
(\%)\end{array}$ & $\begin{array}{l}\text { NPV } \\
(\%)\end{array}$ \\
\hline \multicolumn{6}{|c|}{ Weaning parameters for the PS $5 / 5$ group } \\
\hline$\Delta \mathrm{tdi} \%$ & 36 & 96 & 64 & 86 & 88 \\
\hline$\Delta \mathrm{tdi} \% \times \mathrm{V}_{\mathrm{T}}$ & 36 & 96 & 64 & 86 & 88 \\
\hline $\begin{array}{l}\text { tdi at } \\
\text { end-expiration }\end{array}$ & 36 & 84 & 18 & 70 & 33 \\
\hline \multicolumn{6}{|c|}{ Weaning parameters for the SB trial group } \\
\hline$\Delta$ tdi $\%$ & 27 & 75 & 100 & 100 & 67 \\
\hline$\Delta \mathrm{tdi} \% \times \mathrm{V}_{\mathrm{T}}$ & 27 & 78 & 100 & 100 & 38 \\
\hline RSBI & 26 & 87 & 33 & 91 & 25 \\
\hline $\begin{array}{l}\text { tdi at } \\
\text { end-expiration }\end{array}$ & 27 & 96 & 33 & 92 & 50 \\
\hline
\end{tabular}

to a PS of $\Delta 5 / 5$ had a median of 5.00 days of ventilator support with an IQR of 4.00. The patients weaned with a SB trial received a median of 5.00 days of ventilator support with an IQR of 8.50 days.

The $\Delta$ tdi $\%$ and tdi end-expiration values for all subjects are shown in figures $2 \mathrm{~A}, \mathrm{~B}$ respectively. Of the 49 patients who were successfully extubated, 43 had a $\Delta$ tdi $\%$ of $\geq 30 \%$. Of the 14 who failed extubation, 10 had a $\Delta$ tdi $\%<30 \%$. The resulting sensitivity and specificity was $88 \%$ and $71 \%$, respectively. The PPV of a $\Delta \mathrm{tdi} \% \geq 30 \%$ for extubation success was $91 \%$ and the NPV of a $\Delta$ tdi $<30 \%$ for extubation failure was $63 \%$ (table 1 ). The area under the ROC curve for $\Delta$ tdi $\%$ was 0.79 (figure $3 \mathrm{~A}$ ) and for tdi end-expiration it was 0.61 (figure $3 \mathrm{~B}$ ).

Four patients failed extubation despite having a $\Delta$ tdi $\% \geq 30 \%$. However, factors unrelated to diaphragm contractility such as the development of congestive heart failure (CHF), fever and acute change in mental status, mucous plugging, and aspiration, precipitated respiratory failure and the need for reintubation in these individuals. These non-mechanical factors lowered the NPV and specificity of $\Delta$ tdi $\%$. The remaining 10 patients who failed extubation had a $\Delta$ tdi $\%<30 \%$. In these individuals extubation failure was attributed to 'respiratory pump' failure in eight. One patient failed because of fluid overload and fever and one because of a new cerebrovascular accident (CVA) and aspiration.

When comparing the subgroups of those weaned using PS and those weaned with a SB trial, $\Delta$ tdi $\%$ predicted extubation success or failure equally well. Of the 25 patients who were successfully extubated at a PS of $\Delta 5 / 5,24$ had a $\Delta$ tdi $\% \geq 30 \%$. Of the 11 patients who failed extubation, 7 had a $\Delta$ tdi $\%$ $<30 \%$. The resulting sensitivity, specificity, PPV and NPV are listed in table 2 . Of the 24 patients successfully extubated after a SBT, 18 had a $\Delta$ tdi $\% \geq 30 \%$. Of the three patients who failed extubation, all had a $\Delta$ tdi $\%<30 \%$. The resulting sensitivity, specificity, PPV and NPV are listed in table 2. The RSBI was calculated for 27 patients who underwent a SBT. The sensitivity, specificity, PPV and NPV for a RSBI $\geq 105$ are given in table 2 .

We evaluated if measures of tdi at end-expiration or if composite measures of $\Delta \mathrm{tdi} \%$ and breathing pattern would be better predictors of successful extubation than $\Delta$ tdi $\%$ alone (table 1 ). None of these parameters (tdi at end-expiration, $\Delta \mathrm{tdi} \% \times \mathrm{V}_{\mathrm{T}}$ and $\Delta \mathrm{tdi} \% / \mathrm{f})$ enhanced the predictive value of $\Delta \mathrm{tdi} \%$ alone.
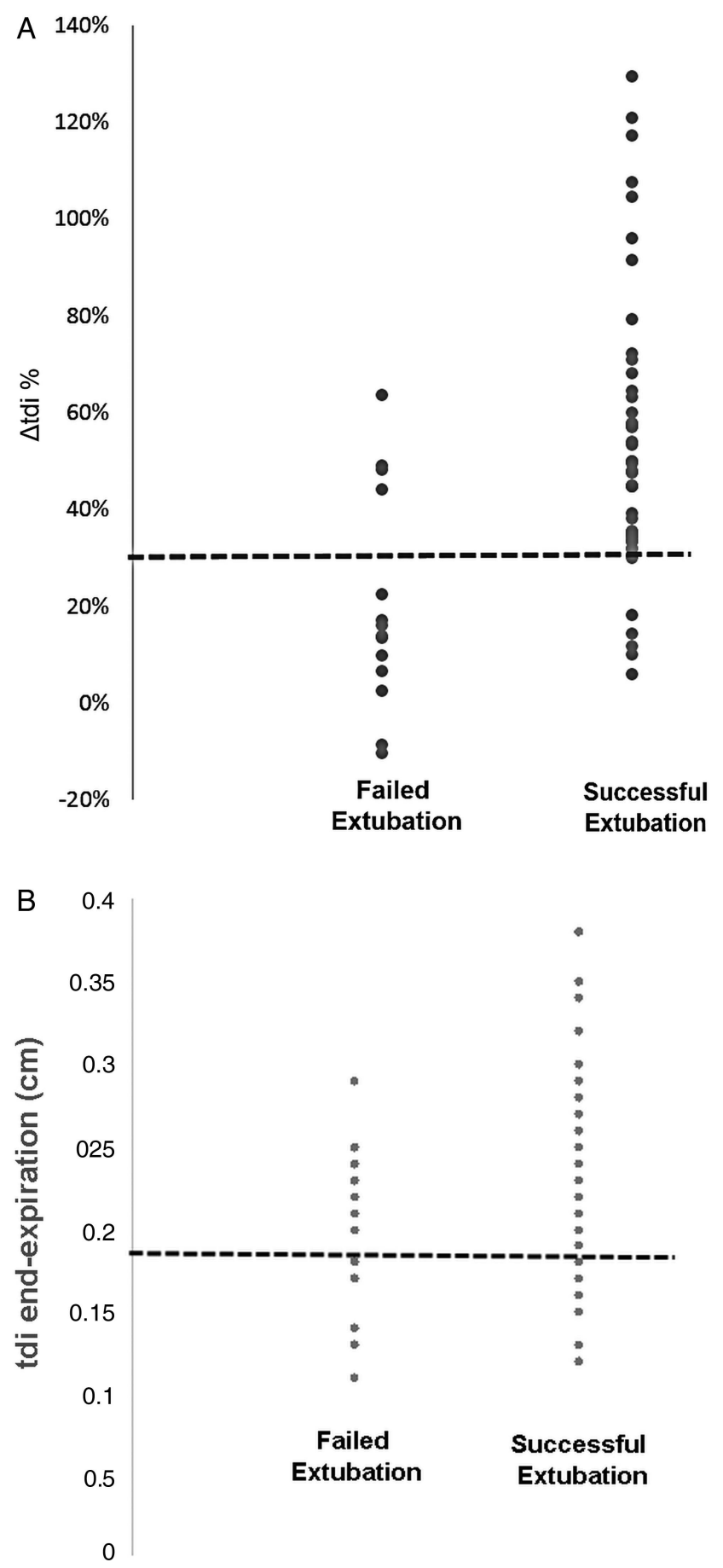

Figure 2 Values for (A) $\Delta$ tdi\%, (B) tdi end-expiration are depicted for all subjects who were successfully extubated or failed extubation. The dashed line represents the cut-off value for $\Delta$ tdi\% $(\geq 30 \%)$ and tdi end-expiration $(\geq 0.17 \mathrm{~cm})$.

\section{DISCUSSION}

Ultrasound is a technology that is increasingly used by intensive care physicians to assist in central line placement and other procedures. ${ }^{26}$ Recently, ultrasound has been used to assess the presence of diaphragm dysfunction postoperatively, ${ }^{27}$ to identify the occurrence of ventilator induced diaphragm injury ${ }^{3}$ and to evaluate diaphragm dome motion during SB weaning trials. ${ }^{16}$ However, the extent to which the diaphragm dome moves during inspiration can be affected by breath size, impedance of neighbouring structures and abdominal compliance. These 

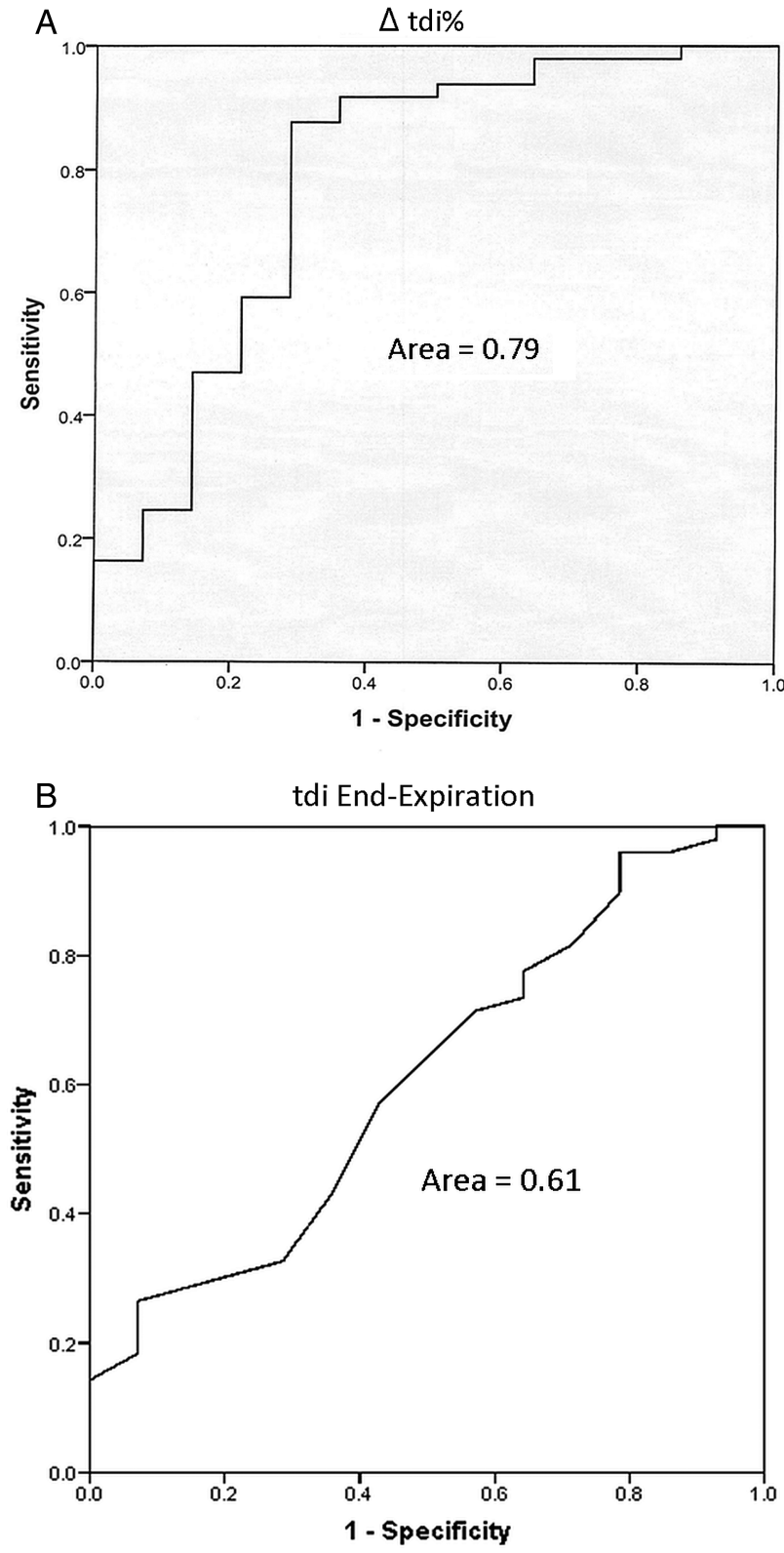

Figure 3 Receiver operating characteristic (ROC) curves for (A) $\Delta$ tdi\%, (B) tdi end-expiration. The area under the curve is expressed as a ratio of the total area.

confounders can be circumvented by visualising the diaphragm muscle itself in the zone of apposition. The present study indicates $\mathrm{B}$ mode ultrasound can be used to predict extubation outcomes during PS and SB weaning trials.

$B$ mode ultrasound measurements of tdi have been correlated with diaphragm strength and muscle shortening. ${ }^{19} 2023$ The volume of diaphragm muscle mass is constant as it contracts. Therefore as it shortens, it thickens and measures of $\Delta$ tdi are inversely related to changes in diaphragm length (ldi) $(\Delta \mathrm{tdi} \approx 1 /$ $\Delta \mathrm{ldi})$. In support of this notion, calculation of $\Delta$ ldi from measures of $\Delta$ tdi in healthy individuals are in the range of what has been measured in humans and the absence of diaphragm thickening has been noted in patients with diaphragm paralysis. ${ }^{20} 28$ Since the diaphragm is the major muscle of inspiration, the presence of diaphragm contraction and shortening should be a prerequisite for successful extubation. The high PPV of $\Delta \mathrm{tdi} \% \geq$
$30 \%$ and our finding that 10 of 14 patients who failed extubation had a $\Delta \mathrm{tdi} \%<30 \%$ is consistent with this concept.

We found that diaphragm contraction, as assessed by $\Delta \mathrm{tdi} \%$ performed better than simultaneous measures of RSBI during SBT. The superiority of $\Delta$ tdi\% to RSBI during SBT may be attributed to the importance of the diaphragm's contribution to $\mathrm{V}_{\mathrm{T}}$. The RSBI is an integrative function of respiratory load and inspiratory muscle capacity. It reflects the function of all inspiratory muscles including the diaphragm, scalenes, parasternal intercostals and accessory inspiratory muscles (sternomastoids and external intercostals). If the diaphragm is failing, the nondiaphragm inspiratory muscles will compensate to preserve $V_{T}$ and the presence of diaphragm weakness may be 'masked' by the increased contribution of the non-diaphragm inspiratory muscles ('rib cage' muscles) to $\mathrm{V}_{\mathrm{T}}$. However, the rib cage muscles are more fatigable and weaker than the diaphragm, and these muscles will not be able to sustain adequate ventilation. ${ }^{29}{ }^{30}$ Accordingly, extubation failure may occur despite an initially acceptable $\mathrm{V}_{\mathrm{T}}$ and RSBI. In this condition, direct measures of diaphragm function using B mode would better predict extubation failure as was the case in 10 of 14 patients who the intensivist judged as ready for extubation using conventional weaning criteria but had a $\Delta$ tdi $\%<30 \%$ and required reintubation.

We evaluated tdi at end-expiration and combined measures of $\Delta$ tdi $\%$ with components of breathing pattern $\left(V_{T}\right.$ and $\left.f\right)$. We found that the ROC for tdi end-expiration was less than that for $\Delta$ tdi $\%$ alone $(0.79$ for $\Delta$ tdi $\%$ and 0.61 for tdi end-expiration). The failure of this model to improve extubation predictions may be related to the variability of tdi among individuals ${ }^{24} 25$ or the presence of ventilator induced diaphragm atrophy in some but not other patients. ${ }^{3}$ We reasoned that the product of $\Delta$ tdi $\%$ and $\mathrm{V}_{\mathrm{T}}$ may be a better predictor of extubation success than $\Delta$ tdi $\%$ alone. Diaphragm shortening contributes to the majority of volume change during inspiration. For a given degree of diaphragm shortening, an 'efficient' diaphragm will yield a greater tidal volume and lower breathing frequency than a diaphragm contracting at a mechanical disadvantage. However, we found that incorporating either $\mathrm{V}_{\mathrm{T}}$ or $\mathrm{f}$ with $\Delta$ tdi $\%$ did not improve extubation predictions.

One limitation of our study is that measures of $\Delta \mathrm{tdi} \%$ were not performed immediately before extubation. It is possible that some patients with a $\Delta$ tdi $\%<30 \% 12-36 \mathrm{~h}$ prior to extubation and were successfully extubated may have had a $\Delta$ tdi $\% \geq 30 \%$ immediately prior to extubation. The converse is also true. A prospective study designed with measures of $\Delta$ tdi $\%$ immediately prior to extubation is needed to address the above limitation. Another possible limitation would be variability of end-expiratory lung volume. Although absolute values of end-expiratory lung volume were not measured, we used a flowmeter to identify end-expiration and end-inspiration and it is unlikely that functional residual capacity (FRC) changed significantly during the brief period when $\Delta$ tdi $\%$ was measured. Finally, our study also only evaluated $\Delta \mathrm{tdi} \%$ for the right hemidiaphragm. We chose to only evaluate the right hemidiaphragm because the acoustic window provided by the liver makes it easier to make the measurement in the right ZAP and we wanted to evaluate a weaning index that would be practical and easy for intensivists to implement.

We conclude that ultrasound measures of diaphragm muscle thickening may predict extubation success or failure with PS and SB weaning trials and that this method may be especially helpful in reducing the number of failed extubations. This measure of diaphragm function can be performed at the bedside, requires 
no special effort by the patient, and can be used during either PS or SB trials. The ubiquitous presence of ultrasound equipment in intensive care units indicates that there need not be additional capital equipment expenditures. The portability and availability of ultrasound make measures of tdi ideally suited for incorporation into the intensivist's decision-making process to complement the complete assessment of the patient in evaluating extubation outcome.

Contributors FDM is the guarantor of the content of the manuscript, including the data and analysis. ED has made substantial contributions to acquisition of data, data analysis, data interpretation and drafting the manuscript EJG has made substantial contributions to design, data interpretation, and provided critical revisions for important intellectual content. JMS has made substantial contributions to design, data interpretation, and provided critical revisions for important intellectual content.

\section{Competing interests None.}

Ethics approval Memorial Hospital of Rhode Island IRB and Rhode Island Hospital IRB.

Provenance and peer review Not commissioned; externally peer reviewed.

\section{REFERENCES}

1 Esteban A, Anzueto A, Frutos F, et al. Characteristics and outcomes in adult patients receiving mechanical ventilation: a 28-day international study. JAMA 2002;287:345-55.

2 Funk GC, Anders S, Breyer MK, et al. Incidence and outcome of weaning from mechanical ventilation according to new categories. Eur Respir J 2010;35:88-94

3 Grosu HB, Lee YI, Lee J, et al. Diaphragm muscle thinning in patients who are mechanically ventilated. Chest 2012;142:1455-60.

4 Hudson MB, Smuder AJ, Nelson WB, et al. Both high level pressure support ventilation and controlled mechanical ventilation induce diaphragm dysfunction and atrophy. Crit Care Med 2012;40:1254-60.

5 Vassilakopoulos T. Ventilator-induced diaphragm dysfunction: the clinical relevance of animal models. Intensive Care Med 2008;34:7-16.

6 Levine $\mathrm{S}$, Nguyen $\mathrm{T}$, Taylor $\mathrm{N}$, et al. Rapid disuse atrophy of diaphragm fibers in mechanically ventilated humans. N Engl J Med 2008;358:1327-35.

7 Ely EW, Baker AM, Evans GW, et al. The prognostic significance of passing a daily screen of weaning parameters. Intensive Care Med 1999;25:581-7.

8 Stroetz RW, Hubmayr RD. Tidal volume maintenance during weaning with pressure support. Am J Respir Crit Care Med 1995;152:1034-40.

9 Krieger BP, Ershowsky PF, Becker DA, et al. Evaluation of conventional criteria for predicting successful weaning from mechanical ventilatory support in elderly patients. Critical Care Med 1989;17:858-61.

10 Nemer SN, Barbas CS, Caldeira JB, et al. Evaluation of maximal inspiratory pressure, tracheal airway occlusion pressure, and its ratio in the weaning outcome. J Crit Care 2009;24:441-6.
11 Conti G, Montini L, Pennisi MA, et al. A prospective, blinded evaluation of indexes proposed to predict weaning from mechanical ventilation. Intensive Care Med 2004;30:830-6.

12 Yang $\mathrm{KL}$, Tobin MJ. A prospective study of indexes predicting the outcome of trials of weaning from mechanical ventilation. N Engl J Med 1991;324:1445-50.

13 Lee KH, Hui KP, Chan TB, et al. Rapid shallow breathing (frequency-tidal volume ratio) did not predict extubation outcome. Chest 1994;105:540-3.

14 Houston JG, Morris AD, Howie CA, et al. Technical report: quantitative assessment of diaphragmatic movement-a reproducible method using ultrasound. Clin Radio/ 1992:46:405-7.

15 Gerscovich EO, Cronan M, McGahan JP, et al. Ultrasonographic evaluation of diaphragmatic motion. J Ultrasound Med 2001;20:597-604.

16 Jiang JR, Tsai TH, Jerng JS, et al. Ultrasonographic evaluation of liver/spleen movements and extubation outcome. Chest 2004;126:179-85.

17 Kim WY, Suh HJ, Hong SB, et al. Diaphragm dysfunction assessed by ultrasonography: influence on weaning from mechanical ventilation. Critical Care Med 2011;39:2627-30.

18 Miller WT, Talman EA. Subphrenic abscess. Am J Roentgenol Radium Ther Nucl Med 1967:101:961-9.

19 Cohn D, Benditt JO, Eveloff S, et al. Diaphragm thickening during inspiration. J Appl Physiol 1997:83:291-6.

20 Gottesman E, McCool FD. Ultrasound evaluation of the paralyzed diaphragm. Am J Respir Crit Care Med 1997;155:1570-4.

21 Summerhill EM, El-Sameed YA, Glidden TJ, et al. Monitoring recovery from diaphragm paralysis with ultrasound. Chest 2008:133:737-43.

22 McCool FD, Tzelepis GE. Dysfunction of the diaphragm. N Engl J Med 2012;366:932-42.

23 McCool FD, Conomos P, Benditt J0, et al. Maximal inspiratory pressures and dimensions of the diaphragm. Am J Respir Crit Care Med 1997;155:1329-34.

$24 \mathrm{McCool}$ FD, Benditt JO, Conomos P, et al. Variability of diaphragm structure among healthy individuals. Am J Respir Crit Care Med 1997;155:1323-8.

25 Rehan VK, Laiprasert J, Wallach $\mathrm{M}$, et al. Diaphragm dimensions of the healthy preterm infant. Pediatrics 2001;108:E91.

26 Matamis D, Soilemezi E, Tsagourias M, et al. Sonographic evaluation of the diaphragm in critically ill patients. Technique and clinical applications. Intensive Care Med 2013:39:801-10.

$27 \mathrm{Kim} \mathrm{SH}$, Na S, Choi JS, et al. An evaluation of diaphragmatic movement by M-mode sonography as a predictor of pulmonary dysfunction after upper abdominal surgery. Anesth Analg 2010;110:1349-54.

28 Rochester DF, Farkas GA, Lu J. Contractility of the in situ diaphragm: assessment based on dimensional analysis. Respiratory Muscles and their Neuromotor Control: Proceedings of an lups Satellite Symposium Held in Los Angeles, California, July 22-24, 1986. New York: Liss, 1987.

29 Hershenson MB, Kikuchi Y, Tzelepis GE, et al. Preferential fatigue of the rib cage muscles during inspiratory resistive loaded ventilation. J Appl Physiol 1989;66:750-4

30 Hershenson MB, Kikuchi Y, Loring SH. Relative strengths of the chest wall muscles. J Appl Physiol 1988;65:852-62. 